\title{
THE CONJECTURE OF NOWICKI ON WEITZENBÖCK DERIVATIONS OF POLYNOMIAL ALGEBRAS
}

\author{
VESSELIN DRENSKY AND LEONID MAKAR-LIMANOV
}

\begin{abstract}
The Weitzenböck theorem states that if $\Delta$ is a linear locally nilpotent derivation of the polynomial algebra $K[Z]=K\left[z_{1}, \ldots, z_{m}\right]$ over a field $K$ of characteristic 0 , then the algebra of constants of $\Delta$ is finitely generated. If $m=2 n$ and the Jordan normal form of $\Delta$ consists of $2 \times 2$ Jordan cells only, we may assume that $K[Z]=K[X, Y]$ and $\Delta\left(y_{i}\right)=x_{i}, \Delta\left(x_{i}\right)=0, i=1, \ldots, n$. Nowicki conjectured that the algebra of constants $K[X, Y]^{\Delta}$ is generated by $x_{1}, \ldots, x_{n}$ and $x_{i} y_{j}-x_{j} y_{i}, 1 \leq i<j \leq n$. Recently this conjecture was confirmed in the Ph.D. thesis of Khoury, and also by Derksen. In this paper we give an elementary proof of the conjecture of Nowicki. Then we find a very simple system of defining relations of the algebra $K[X, Y]^{\Delta}$ which corresponds to the reduced Gröbner basis of the related ideal with respect to a suitable admissible order, and present an explicit basis of $K[X, Y]^{\Delta}$ as a vector space.
\end{abstract}

\section{INTRODUCTION}

Let $K$ be a field of characteristic 0 and let $K[Z]=K\left[z_{1}, \ldots, z_{m}\right]$ be the polynomial $K$-algebra in $m$ variables. A linear operator $\Delta$ of $K[Z]$ is called a derivation if $\Delta(u v)=\Delta(u) v+u \Delta(v)$ for all $u, v \in K[Z]$. The derivation $\Delta$ is locally nilpotent if for each $u \in K[Z]$ there exists a $d \geq 1$ such that $\Delta^{d}(u)=0$. Locally nilpotent derivations of polynomial algebras are subjects of active investigation. They play essential role in the study of automorphism group of $K[Z]$, including the generation of Aut $K[x, y]$ by tame automorphisms, the Jacobian conjecture, in invariant theory, Fourteenth Hilbert's problem and other important topics. See the books by Nowicki [N], van den Essen [E], and Freudenburg [F] for details.

The well known theorem of Weitzenböck [W] states that if $\Delta$ is a nilpotent linear operator acting on the $m$-dimensional vector space $K Z=K z_{1} \oplus \cdots \oplus K z_{m}$ and we extend it to a derivation of $K[Z]$, then the algebra $K[Z]^{\Delta}$ of constants of $\Delta$, i.e., the kernel of $\Delta$ in $K[Z]$, is a finitely generated algebra. We call $\Delta$, which is a locally nilpotent derivation, a Weitzenböck derivation. A modern proof of the theorem of Weitzenböck is given by Seshadri [S], with further simplification by Tyc T], see also [N, $\mathrm{F}]$.

Up to a change of the basis of the vector space $K Z$, Weitzenböck derivations are determined by their Jordan normal form. Each Jordan cell is an upper triangular matrix with zero diagonal. Hence, for each dimension $m$ it is sufficient to consider a finite number of Weitzenböck derivations $\Delta$. There are algorithms which find a set of generators of $K[Z]^{\Delta}$ for a given $\Delta$. Nevertheless from computational point

2000 Mathematics Subject Classification. 13N15; 13A50; 13P10; 14E07.

The research of the first author was partially supported by Grant MI-1503/2005 of the Bulgarian National Science Fund.

The work of the second author was partially supported by an NSA grant. 
of view it is difficult to find explicitly such a system. In particular, no upper bound for the degree of the generators is known.

The algebra of constants of $\Delta$ coincides with the algebra of invariants of the linear operator

$$
\exp (\Delta)=1+\frac{\Delta}{1 !}+\frac{\Delta^{2}}{2 !}+\cdots
$$

and $K[Z]^{\Delta}$ may be studied also with methods of invariant theory. In particular, when we find the generators of the algebra of invariants of $\exp (\Delta)$, this is stated as the First fundamental theorem of the invariants of $\exp (\Delta)$. When we find the defining relations, this is the Second fundamental theorem.

When the Jordan normal form of the Weitzenböck derivation $\Delta$ consists of $2 \times 2$ Jordan cells only, we may assume that

$$
\begin{aligned}
K[Z]=K[X, Y] & =K\left[x_{1}, \ldots, x_{n}, y_{1}, \ldots, y_{n}\right], \\
\Delta & =\sum_{i=1}^{n} x_{i} \frac{\partial}{\partial y_{i}}
\end{aligned}
$$

and hence the action of $\Delta$ is defined by

$$
\Delta\left(x_{i}\right)=0, \quad \Delta\left(y_{i}\right)=x_{i}, \quad i=1, \ldots, n .
$$

Till the end of the paper we shall consider such derivations only. Nowicki [N] conjectured that $K[X, Y]^{\Delta}$ is generated by $x_{1}, \ldots, x_{n}$ and the determinants

$$
u_{i j}=\left|\begin{array}{ll}
x_{i} & x_{j} \\
y_{i} & y_{j}
\end{array}\right|, \quad 1 \leq i<j \leq n .
$$

The conjecture of Nowicki was confirmed by Khoury in his Ph.D. thesis [K] His proof is very computational and uses essentially Gröbner basis techniques. Another proof was given (but not published yet) by Derksen who combined ideas of the proof of Seshadri [S] of the Weitzenböck theorem with the explicit form of the invariants of the special linear group $S L_{2}(K)$ acting on a direct sum of two-dimensional $S L_{2}(K)$ invariant vector spaces.

The purpose of our paper is to give a new elementary proof of the conjecture of Nowicki. We use easy arguments from undergraduate algebra and a simple induction only. We find also a uniformly looking explicit set of defining relations of the algebra of constants $K[X, Y]^{\Delta}$ which corresponds to the reduced Gröbner basis of the related ideal of $K[X, U]$, where $U=\left\{u_{i j} \mid 1 \leq i<j \leq n\right\}$, as well as a basis of $K[X, Y]^{\Delta}$ as a vector space.

\section{The Conjecture of Nowicki}

We fix the sets of variables

$$
\begin{array}{cc}
X^{\prime}=\left\{x_{1}, \ldots, x_{n-1}\right\}, & Y^{\prime}=\left\{y_{1}, \ldots, y_{n-1}\right\} \\
X=X^{\prime} \cup\left\{x_{n}\right\}, & Y=Y^{\prime} \cup\left\{y_{n}\right\}
\end{array}
$$

and the derivation of $K[X, Y]=K\left[X^{\prime}, Y^{\prime}, x_{n}, y_{n}\right]$

$$
\Delta=\sum_{i=1}^{n} x_{i} \frac{\partial}{\partial y_{i}}
$$


The derivation $\Delta$ acts in the same manner on all $x_{i}$ and $y_{i}$. Hence the $K$-algebra endomorphism $\varphi_{\alpha}$ of $K[X, Y], \alpha=\left(\alpha_{1}, \ldots, \alpha_{n-1}\right) \in K^{n-1}$, defined by

$$
\begin{gathered}
\varphi_{\alpha}\left(x_{i}\right)=x_{i}, \quad \varphi_{\alpha}\left(y_{i}\right)=y_{i}, \quad i=1, \ldots, n-1, \\
\varphi_{\alpha}\left(x_{n}\right)=\alpha_{1} x_{1}+\cdots+\alpha_{n-1} x_{n-1}, \quad \varphi_{\alpha}\left(y_{n}\right)=\alpha_{1} y_{1}+\cdots+\alpha_{n-1} y_{n-1},
\end{gathered}
$$

commutes with $\Delta$. If $f\left(X^{\prime}, Y^{\prime}, x_{n}, y_{n}\right)$ belongs to the algebra of constants $K[X, Y]^{\Delta}$, then

$$
\begin{gathered}
\varphi_{\alpha}(f)=f\left(\varphi_{\alpha}\left(X^{\prime}\right), \varphi_{\alpha}\left(Y^{\prime}\right), \varphi_{\alpha}\left(x_{n}\right), \varphi_{\alpha}\left(y_{n}\right)\right) \\
=f\left(X^{\prime}, Y^{\prime}, \alpha_{1} x_{1}+\cdots+\alpha_{n-1} x_{n-1}, \alpha_{1} y_{1}+\cdots+\alpha_{n-1} y_{n-1}\right) \in K\left[X^{\prime}, Y^{\prime}\right]^{\Delta}
\end{gathered}
$$

for all $\alpha=\left(\alpha_{1}, \ldots, \alpha_{n-1}\right) \in K^{n-1}$.

Lemma 1. Let $n \geq 2$ and let a nonzero polynomial $f=f\left(X^{\prime}, Y^{\prime}, x_{n}, y_{n}\right)$ be homogeneous with respect to $x_{n}, y_{n}$. If

$$
\varphi_{\alpha}(f)=f\left(X^{\prime}, Y^{\prime}, \alpha_{1} x_{1}+\cdots+\alpha_{n-1} x_{n-1}, \alpha_{1} y_{1}+\cdots+\alpha_{n-1} y_{n-1}\right)=0
$$

for an $\alpha=\left(\alpha_{1}, \ldots, \alpha_{n-1}\right) \in K^{n-1} \backslash 0$, then $f$ is divisible by

$$
w_{\alpha}\left(X^{\prime}, Y^{\prime}, x_{n}, y_{n}\right)=\left(\alpha_{1} x_{1}+\cdots+\alpha_{n-1} x_{n-1}\right) y_{n}-\left(\alpha_{1} y_{1}+\cdots+\alpha_{n-1} y_{n-1}\right) x_{n} .
$$

Proof. Let

$$
0 \neq f=a_{p} y_{n}^{p}+a_{p-1} x_{n} y_{n}^{p-1}+\cdots+a_{1} x_{n}^{p-1} y_{n}+a_{0} x_{n}^{p}, \quad a_{i} \in K\left[X^{\prime}, Y^{\prime}\right],
$$

be a polynomial homogeneous in $x_{n}, y_{n}$. Then

$$
\begin{gathered}
\left(\alpha_{1} x_{1}+\cdots+\alpha_{n-1} x_{n-1}\right) f=a_{p} w_{\alpha} y_{n}^{p-1} \\
+a_{p}\left(\alpha_{1} y_{1}+\cdots+\alpha_{n-1} y_{n-1}\right) x_{n} y_{n}^{p-1}+\left(\alpha_{1} x_{1}+\cdots+\alpha_{n-1} x_{n-1}\right) x_{n} g,
\end{gathered}
$$

where $g=a_{p-1} y_{n}^{p-1}+a_{p-2} x_{n} y_{n}^{p-2}+\cdots+a_{1} x_{n}^{p-2} y_{n}+a_{0} x_{n}^{p-1}$. Since $\varphi_{\alpha}\left(a_{p}\left(\alpha_{1} y_{1}+\right.\right.$ $\left.\left.\cdots+\alpha_{n-1} y_{n-1}\right) y_{n}^{p-1}+g\right)=0$ we can assume by induction on the degree of a polynomial relative to $x_{n}, y_{n}$ that $w_{\alpha}$ divides $a_{p}\left(\alpha_{1} y_{1}+\cdots+\alpha_{n-1} y_{n-1}\right) y_{n}^{p-1}+g$ (the base of induction for the degree equal to zero is obviously correct) and so $w_{\alpha}$ divides $\left(\alpha_{1} x_{1}+\cdots+\alpha_{n-1} x_{n-1}\right) f$.

The polynomial $w_{\alpha}\left(X^{\prime}, Y^{\prime}, x_{n}, y_{n}\right)$ is irreducible in $K\left[X^{\prime}, Y^{\prime}, x_{n}, y_{n}\right]$. Since $\alpha_{1} x_{1}+$ $\cdots+\alpha_{n-1} x_{n-1}$ does not depend on $x_{n}, y_{n}$, we conclude that $w_{\alpha}\left(X^{\prime}, Y^{\prime}, x_{n}, y_{n}\right)$ divides $f\left(X^{\prime}, Y^{\prime}, x_{n}, y_{n}\right)$ in $K\left[X^{\prime}, Y^{\prime}, x_{n}, y_{n}\right]$.

Corollary 2. If $\varphi_{\alpha}(f)=0$ for all $\alpha=\left(\alpha_{1}, \ldots, \alpha_{n-1}\right) \in K^{n-1} \backslash 0$, then $f=0$ if $n>2$ and $f$ is divisible by $u_{12}=x_{1} y_{2}-x_{2} y_{1}$ if $n=2$.

Explanation. If $n>2$ then $f$ is divisible by $\left(x_{1}+\alpha_{2} x_{2}\right) y_{n}-\left(y_{1}+\alpha_{2} y_{2}\right) x_{n}$ where $\alpha_{2}$ is any element of $K$. So $f$ is divisible by infinitely many pairwise non-proportional irreducible polynomials. This is of course impossible if $f \neq 0$. If $n=2$ then by Lemma 1, $f$ is divisible by $u_{12}$ and in this case all $w_{\alpha}$ with non-zero $\alpha$ are proportional to $u_{12}$.

Theorem 3. Let $X=\left\{x_{1}, \ldots, x_{n}\right\}, Y=\left\{y_{1}, \ldots, y_{n}\right\}, n \geq 1$, and let $\Delta$ be the Weitzenböck derivation of $K[X, Y]$ defined by

$$
\Delta=\sum_{i=1}^{n} x_{i} \frac{\partial}{\partial y_{i}}
$$

The algebra of constants $K[X, Y]^{\Delta}$ is generated by

$$
x_{i}, i=1, \ldots, n, \quad u_{i j}=x_{i} y_{j}-x_{j} y_{i}, 1 \leq i<j \leq n .
$$


Proof. Let

$$
f=f\left(X^{\prime}, Y^{\prime}, x_{n}, y_{n}\right) \in K[X, Y]^{\Delta} .
$$

For a monomial $v \in K[X, Y]$ which is of degree $\left(d_{1}, d_{2}\right)$ with respect to $(X, Y)$, i.e., of degree $d_{1}$ in $X$ and $d_{2}$ in $Y$, the image $\Delta(v)$ is of degree $\left(d_{1}+1, d_{2}-1\right)$. Hence, if $f \in K[X, Y]^{\Delta}$, then the homogeneous in $(X, Y)$ components of $f$ are also in $K[X, Y]^{\Delta}$ and we may assume that $f$ is homogeneous in $(X, Y)$. Similarly, if a monomial $u$ is of total degree $p$ in $x_{n}, y_{n}$, the same is true for $\Delta(u)$. Again, we may assume that $f$ is homogeneous in $x_{n}, y_{n}$. We shall prove the theorem by induction on $n$ and on the total degree in $x_{n}, y_{n}$. If $n=1$, then $K\left[x_{1}, y_{1}\right]^{\Delta}=K\left[x_{1}\right]$. We assume that $n>1$ and $K\left[X^{\prime}, Y^{\prime}\right]^{\Delta}$ is generated by $X^{\prime}$ and $U^{\prime}=\left\{u_{i j} \mid 1 \leq i<\right.$ $j \leq n-1\}$. Since $\operatorname{deg}_{X}\left(u_{i j}\right)=\operatorname{deg}_{Y}\left(u_{i j}\right)=1$ and $1=\operatorname{deg}_{X}\left(x_{i}\right)>\operatorname{deg}_{Y}\left(x_{i}\right)=0$, the homogeneous in $(X, Y)$ elements $v \in K\left[X^{\prime}, Y^{\prime}\right]^{\Delta}$ satisfy $\operatorname{deg}_{X^{\prime}}(v) \geq \operatorname{deg}_{Y^{\prime}}(v)$. If $\operatorname{deg}_{x_{n}, y_{n}}(f)=0$, then $f=f\left(X^{\prime}, Y^{\prime}\right) \in K\left[X^{\prime}, Y^{\prime}\right]^{\Delta}$. Hence we may consider the case $\operatorname{deg}_{x_{n}, y_{n}}(f)>0$. We write $f$ in the form

$$
f=\left(a_{p} y_{n}^{p}+a_{p-1} x_{n} y_{n}^{p-1}+\cdots+a_{1} x_{n}^{p-1} y_{n}+a_{0} x_{n}^{p}\right) x_{n}^{q},
$$

where $a_{j}=a_{j}\left(X^{\prime}, Y^{\prime}\right) \in K\left[X^{\prime}, Y^{\prime}\right]$ and $a_{p} \neq 0$. Since both $f$ and $x_{n}^{q}$ belong to $K[X, Y]^{\Delta}$, the same holds for $f / x_{n}^{q}$ and we may assume that $q=0$. The next observation is that $a_{p} \in K\left[X^{\prime}, Y^{\prime}\right]^{\Delta}$ because

$$
0=\Delta(f)=\Delta\left(a_{p}\right) y_{n}^{p}+\left(p a_{p}+\Delta\left(a_{p-1}\right)\right) x_{n} y_{n}^{p-1}+\cdots+\left(a_{1}+\Delta\left(a_{0}\right)\right) x_{n}^{p} .
$$

Hence $a_{p}$ has the form

$$
a_{p}=\sum x_{s_{1}} \cdots x_{s_{k}} b_{s}\left(U^{\prime}\right) .
$$

If $\varphi_{\alpha}(f)=0$ for all $\alpha \in K^{n-1}$, then Corollary 2 gives that $n=2$ and $f$ is divisible by $u_{12}$. Hence $f_{1}=f / u_{12}$ also belongs to $K[X, Y]^{\Delta}$. Since $\operatorname{deg}_{x_{2}, y_{2}}\left(f_{1}\right)=$ $\operatorname{deg}_{x_{2}, y_{2}}(f)-1$, we apply inductive arguments and conclude that $f \in K\left[x_{1}, x_{2}, u_{12}\right]$. Now we consider the case when $\varphi_{\alpha}(f) \neq 0$ for some $\alpha \in K^{n-1}$. The operators $\varphi_{\alpha}$ and $\Delta$ commute, so we have that $\varphi_{\alpha}(f) \in K\left[X^{\prime}, Y^{\prime}\right]^{\Delta}$. Also clearly

$$
\operatorname{deg}_{X}(f)=\operatorname{deg}_{X^{\prime}}\left(\varphi_{\alpha}(f)\right) \geq \operatorname{deg}_{Y^{\prime}}\left(\varphi_{\alpha}(f)\right)=\operatorname{deg}_{Y}(f) .
$$

Hence

$$
\begin{aligned}
& \operatorname{deg}_{X}(f)=\operatorname{deg}_{X^{\prime}}\left(a_{p}\right) \geq \operatorname{deg}_{Y}(f)=p+\operatorname{deg}_{Y^{\prime}}\left(a_{p}\right) \text { and } \\
& f=\sum x_{s_{1}} \cdots x_{s_{p}} c_{s}\left(X^{\prime}, U^{\prime}\right) y_{n}^{p}+\sum_{i=0}^{p-1} a_{i}\left(X^{\prime}, Y^{\prime}\right) x_{n}^{p-i} y_{n}^{i} .
\end{aligned}
$$

We rewrite $x_{s_{j}} y_{n}$ in the form

$$
x_{s_{j}} y_{n}=\left(x_{s_{j}} y_{n}-x_{n} y_{s_{j}}\right)+x_{n} y_{s_{j}}=u_{s_{j} n}+x_{n} y_{s_{j}}
$$

and obtain

$$
x_{s_{1}} \cdots x_{s_{p}} y_{n}^{p}=\prod_{j=1}^{p}\left(u_{s_{j} n}+x_{n} y_{s_{j}}\right)=\prod_{j=1}^{p} u_{s_{j} n}+x_{n} g\left(X^{\prime}, Y^{\prime}, x_{n}, y_{n}\right)
$$

for some $g\left(X^{\prime}, Y^{\prime}, x_{n}, y_{n}\right) \in K\left[X^{\prime}, Y^{\prime}, x_{n}, y_{n}\right]$. Hence

$$
f=\sum c_{s}\left(X^{\prime}, U^{\prime}\right)\left(\prod_{j=1}^{p} u_{s_{j} n}+x_{n} g\left(X^{\prime}, Y^{\prime}, x_{n}, y_{n}\right)\right)+\sum_{i=0}^{p-1} a_{i}\left(X^{\prime}, Y^{\prime}\right) x_{n}^{p-i} y_{n}^{i}
$$




$$
=\sum c_{s}\left(X^{\prime}, U^{\prime}\right) \prod_{j=1}^{p} u_{s_{j} n}+x_{n} f_{1}\left(X^{\prime}, Y^{\prime}, x_{n}, y_{n}\right)
$$

for some $f_{1}\left(X^{\prime}, Y^{\prime}, x_{n}, y_{n}\right) \in K\left[X^{\prime}, Y^{\prime}, x_{n}, y_{n}\right]$. Since $f, x_{n}, c_{s}\left(X^{\prime}, U^{\prime}\right) \prod_{j=1}^{p} u_{s_{j} n} \in$ $K[X, Y]^{\Delta}$, the same is true for $f_{1}\left(X^{\prime}, Y^{\prime}, x_{n}, y_{n}\right)$ and we apply induction on the degree of $f_{1}$ in $x_{n}, y_{n}$. This completes the proof of the theorem.

\section{DeFining RELATions}

In this section we shall show that the defining relations of the algebra of constants $K[X, Y]^{\Delta}$, with respect to the generators $x_{i}, u_{i j}=x_{i} y_{j}-x_{j} y_{i}$ from the conjecture of Nowicki, consists of the relations

$$
\begin{gathered}
r(i, j, k, l)=u_{i j} u_{k l}-u_{i k} u_{j l}+u_{i l} u_{j k}=0, \quad 1 \leq i<j<k<l \leq n, \\
s(i, j, k)=x_{i} u_{j k}-x_{j} u_{i k}+x_{k} u_{i j}=0, \quad 1 \leq i<j<k \leq n .
\end{gathered}
$$

Our proof will give that the elements $r(i, j, k, l)$ and $s(i, j, k)$ form the reduced Gröbner basis of the corresponding ideal of $K[X, U], U=\left\{u_{i j} \mid 1 \leq i<j \leq n\right\}$, with respect to a suitable admissible order. This provides also a basis of $K[X, Y]^{\Delta}$ as a vector space.

It will be convenient to identify the generator $u_{i j} \in U$ with the open interval $(i, j)$ on the real line and define the interval length of $u_{i j}$ by $\left|u_{i j}\right|=j-i$. We say that $u_{i j}$ and $u_{k l}$ intersect each other if the intervals $(i, j)$ and $(k, l)$ have a nonempty intersection and are not contained in each other. This means that one of the inequalities $i<k<j<l$ and $k<i<l<j$ holds. We say also that $u_{i j}$ covers $x_{k}$ if $k$ belongs to the open interval $(i, j)$.

We summarize the necessary background on Gröbner bases. For more details we refer for example to the book by Adams and Loustaunau $[\mathrm{AL}$.

Let $Z=\left\{z_{1}, \ldots, z_{m}\right\}$ be a set of variables. The linear ordering $\succ$ on the set of monomials $[Z]$ is admissible, if it satisfies the descending chain condition and $u \succ v$ for $u, v \in[Z]$ imlies $u w \succ v w$ for all $w \in[Z]$. Every nonzero polynomial $f(Z)$ of $K[Z]$ can be written as

$$
f=\beta_{1} v_{1}+\beta_{2} v_{2}+\cdots+\beta_{k} v_{k}, \quad 0 \neq \beta_{j} \in K
$$

where $v_{1} \succ v_{2} \succ \cdots \succ v_{k}$. We denote by $\bar{f}$ the leading monomial $v_{1}$ of $f$ in [Z].

Let $J$ be an ideal of $K[Z]$ and let $I(J)$ be the set of leading monomials of $J$. A generating set $G$ of $J$ is called a Gröbner basis of $J$ (with respect to the fixed admissible order on $[Z]$ ) if for any $f \in J$ there exists an $f_{i} \in G$ such that $\bar{f}$ is divisible by $\overline{f_{i}}$. Equivalently, the set $I(G)$ of leading monomials of $G$ generates the semigroup ideal $I(J)$ of $[Z]$. A subset $G$ of $J$ is a Gröbner basis of $J$ if and only if it has the following property. The set of normal monomials of $[Z]$ with respect to $G$, i.e., the monomials which are not divisible by an element of $I(G)$, forms a $K$-basis of the factor algebra $K[Z] / J$. The set of normal monomials with respect to $G$ spans $K[Z] / J$ for any subset $G$ of $J$. Hence $G \subset J$ is a Gröbner basis of $J$ if and only if the set of normal monomials with respect to $G$ is linearly independent in the factor algebra $K[Z] / J$. The Gröbner basis $G$ is reduced if the monomials $v_{i}$ participating in each $f_{j} \in G$ are not divisible by the leading monomials of $G \backslash\left\{f_{j}\right\}$.

It is easy to see that the following ordering is admissible. We believe that it may be applied also to other problems. 
Definition 4. We order the monomials of $K[X, U]$ first by the degree in $X$ and $U$, then by the total interval length of the participating variables $u_{i j}$ and finally lexicographically, as follows.

Let

$$
v=x_{i_{1}} \cdots x_{i_{c}} u_{j_{1} k_{1}} \cdots u_{j_{d} k_{d}}
$$

where $i_{1} \leq \cdots \leq i_{c}, j_{1} \leq \cdots \leq j_{d}$ and $k_{a} \leq k_{a+1}$ if $j_{a}=j_{a+1}$, and

$$
v^{\prime}=x_{i_{1}^{\prime}} \cdots x_{i_{c^{\prime}}^{\prime}} u_{j_{1}^{\prime} k_{1}^{\prime}} \cdots u_{j_{d^{\prime}}^{\prime} k_{d^{\prime}}^{\prime}}
$$

with similar restrictions on $i_{a}^{\prime}, j_{b}^{\prime}, k_{b}^{\prime}$.

We define $v \succ v^{\prime}$ if

(i) $c>c^{\prime}$ (the degree of $v$ in $X$ is bigger than the degree of $v^{\prime}$ in $X$ );

(ii) $c=c^{\prime}$ and $d>d^{\prime}$ (the degree of $v$ in $U$ is bigger than the degree of $v^{\prime}$ in $U$ );

(iii) $c=c^{\prime}, d=d^{\prime}$ and

$$
\sum_{b=1}^{d}\left|u_{j_{b} k_{b}}\right|>\sum_{b=1}^{d}\left|u_{j_{b}^{\prime} k_{b}^{\prime}}\right|
$$

(the total interval length of $v$ is bigger than that of $v^{\prime}$ );

(iv) $c=c^{\prime}, d=d^{\prime}$,

$$
\sum_{b=1}^{d}\left|u_{j_{b} k_{b}}\right|=\sum_{b=1}^{d}\left|u_{j_{b}^{\prime} k_{b}^{\prime}}\right|
$$

and $\omega \succ \omega^{\prime}$ for the $(c+2 d)$-tuples

$$
\omega=\left(i_{1}, \ldots, i_{c}, j_{1}, \ldots, j_{d}, k_{1}, \ldots, k_{d}\right), \quad \omega^{\prime}=\left(i_{1}^{\prime}, \ldots, i_{c}^{\prime}, j_{1}^{\prime}, \ldots, j_{d}^{\prime}, k_{1}^{\prime}, \ldots, k_{d}^{\prime}\right),
$$

where $\left(a_{1}, \ldots, a_{p}\right) \succ\left(b_{1}, \ldots, b_{p}\right)$ if $a_{1}=b_{1}, \ldots, a_{e}=b_{e}, a_{e+1}<b_{e+1}$ for some $e$.

We call this ordering degree-interval length-lexicographic order (DILL order) of $K[X, U]$.

Theorem 5. Let $X=\left\{x_{1}, \ldots, x_{n}\right\}, Y=\left\{y_{1}, \ldots, y_{n}\right\}, n \geq 1$, and let $\Delta$ be the Weitzenböck derivation of $K[X, Y]$ defined by

$$
\Delta=\sum_{i=1}^{n} x_{i} \frac{\partial}{\partial y_{i}}
$$

(i) The algebra of constants has the presentation

$$
K[X, Y]^{\Delta} \cong K[X, U] /(R, S),
$$

where $X=\left\{x_{i} \mid i=1, \ldots, n\right\}, U=\left\{u_{i j} \mid 1 \leq i<j \leq n\right\}$ and the ideal $(R, S)$ is generated by

$$
\begin{gathered}
R=\left\{r(i, j, k, l)=u_{i j} u_{k l}-u_{i k} u_{j l}+u_{i l} u_{j k} \mid 1 \leq i<j<k<l \leq n\right\}, \\
S=\left\{s(i, j, k)=x_{i} u_{j k}-x_{j} u_{i k}+x_{k} u_{i j} \mid 1 \leq i<j<k \leq n\right\} .
\end{gathered}
$$

(ii) The set $R \cup S$ is the reduced Gröbner basis of the ideal $(R, S)$ with respect to the DILL order of $K[X, U]$.

(iii) As a vector space $K[X, Y]^{\Delta}$ has a basis consisting of all products

$$
x_{i_{1}} \cdots x_{i_{c}} u_{j_{1} k_{1}} \cdots u_{j_{d} k_{d}}
$$

such that the generators $u_{j_{p} k_{p}}$ and $u_{j_{q} k_{q}}$ do not intersect each other and $u_{j_{p} k_{p}}$ does not cover $x_{i_{t}}$ for any $p, q, t$. 
Proof. Let $\pi: K[X, U] \rightarrow K[X, Y]^{\Delta}$ be the homomorphism defined by

$$
\pi\left(x_{i}\right)=x_{i}, \quad \pi\left(u_{j k}\right)=x_{j} y_{k}-x_{k} y_{j}
$$

and let $J=\operatorname{Ker}(\pi) \subset K[X, U]$. We shall prove the following:

(1) The ideal $J$ contains $R$ and $S$;

(2) The set of normal monomials with respect to $R \cup S$ coincides with the set of all products

$$
x_{i_{1}} \cdots x_{i_{c}} u_{j_{1} k_{1}} \cdots u_{j_{d} k_{d}}
$$

such that the generators $u_{j_{p} k_{p}}$ and $u_{j_{q} k_{q}}$ do not intersect each other and $u_{j_{p} k_{p}}$ does not cover $x_{i_{t}}$ for any $p, q, t$;

(3) The images of these normal monomials under $\pi$ are linearly independent in $K[X, Y]$.

Since $K[X, Y]$ contains $K[X, Y]^{\Delta}=\pi(K[X, U]) \cong K[X, U] / J$, the statement (3) implies that the images of normal monomials under $\pi$ are linearly independent in $K[X, U] / J$ and so, as we mentioned above, $R \cup S$ is a Gröbner basis of $J=\operatorname{Ker}(\pi)$. We also check that this basis is reduced.

Step 1: It is easy to verify directly that $\pi(s(i, j, k))=\pi(r(i, j, k, l))=0$. Also

$$
\pi(s(i, j, k))=\operatorname{det}\left(\begin{array}{ccc}
x_{i} & x_{j} & x_{k} \\
x_{i} & x_{j} & x_{k} \\
y_{i} & y_{j} & y_{k}
\end{array}\right)
$$

expended relative to the first row and

$$
2 \pi(r(i, j, k, l))=\operatorname{det}\left(\begin{array}{cccc}
x_{i} & x_{j} & x_{k} & x_{l} \\
y_{i} & y_{j} & y_{k} & y_{l} \\
x_{i} & x_{j} & x_{k} & x_{l} \\
y_{i} & y_{j} & y_{k} & y_{l}
\end{array}\right)
$$

expended relative to the first two rows.

Step 2: If $1 \leq i<j<k<l \leq n$ then $\overline{r(i, j, k, l)}=u_{i k} u_{j l}$ since $\left|u_{i k} u_{j l}\right|=$ $\left|u_{i l} u_{j k}\right|>\left|u_{i j} u_{k l}\right|$ and $(i, j, k, l) \succ(i, j, l, k)$. Similarly, if $1 \leq i<j<k \leq n$ then $\overline{s(i, j, k)}=x_{j} u_{i k}$ since $\left|u_{i k}\right|>\left|u_{j k}\right|$ and $\left|u_{i k}\right|>\left|u_{i j}\right|$. So different leading monomials do not divide each other and the set $R \cup S$ is reduced.

Let

$$
v=x_{i_{1}} \cdots x_{i_{c}} u_{j_{1} k_{1}} \cdots u_{j_{d} k_{d}}
$$

be a normal monomial with respect to $R \cup S$. If two variables $u_{i k}$ and $u_{j l}$ intersect each other, i.e., either $1 \leq i<j<k<l \leq n$ or $1 \leq j<i<l<k \leq n$, then their product $u_{i k} u_{j l}$ is the leading monomial of $r(i, j, k, l)$ or $r(k, l, i, j)$ accordingly. Hence in the normal monomial $v$ the participating generators $u_{j_{p} k_{p}}$ and $u_{j_{q} k_{q}}$ do not intersect each other. Again, if $u_{i k}$ covers $x_{j}$, i.e., $i<j<k$, then $x_{j} u_{i k}$ is the leading monomial of $s(i, j, k)$. Hence $u_{j_{p} k_{p}}$ does not cover $x_{i_{t}}$ in the normal monomial $v$ for any $p, q, t$.

Step 3: We want to show that the images of the normal monomials under $\pi$ are linearly independent in $K[X, Y]$.

Introduce the following ordering on the monomials of $K[X, Y]$ :

$$
x_{1}^{a_{1}} y_{1}^{b_{1}} \cdots x_{n}^{a_{n}} y_{n}^{b_{n}}>x_{1}^{a_{1}^{\prime}} y_{1}^{b_{1}^{\prime}} \cdots x_{n}^{a_{n}^{\prime}} y_{n}^{b_{n}^{\prime}},
$$

if $\left(a_{1}, b_{1}, \ldots, a_{n}, b_{n}\right)>\left(a_{1}^{\prime}, b_{1}^{\prime}, \ldots, a_{n}^{\prime}, b_{n}^{\prime}\right)$ lexicographically, i.e., either $a_{1}>a_{1}^{\prime}$ or $a_{1}=a_{1}^{\prime}, b_{1}=b_{1}^{\prime}, \ldots, b_{k}=b_{k}^{\prime}$, and either $a_{k+1}>a_{k+1}^{\prime}$ or $a_{k+1}=a_{k+1}^{\prime}, b_{k+1}>b_{k+1}^{\prime}$ for some $k$. The leading monomial of $\pi\left(u_{j k}\right)=x_{j} y_{k}-x_{k} y_{j}, j<k$, in $K[X, Y]$ 
is lead $\left(u_{j k}\right)=x_{j} y_{k}$ since $x_{j}^{1} y_{j}^{0} x_{k}^{0} y_{k}^{1}>x_{j}^{0} y_{j}^{1} x_{k}^{1} y_{k}^{0}$ and the leading monomial of $w=$ $x_{i_{1}} \cdots x_{i_{c}} u_{j_{1} k_{1}} \cdots u_{j_{d} k_{d}}$ is

$$
\operatorname{lead}(w)=x_{i_{1}} \cdots x_{i_{c}} x_{j_{1}} y_{k_{1}} \cdots x_{j_{d}} y_{k_{d}}
$$

so $\operatorname{deg}_{Y}(\pi(w))=\operatorname{deg}_{U}(w)$.

We shall prove by induction on $\operatorname{deg}_{Y}(\pi(w))$ that a normal monomials $w \in$ $K[X, U]$ is uniquely determined by its leading monomial lead $(w)$.

If $\operatorname{deg}_{Y}(\pi(w))=0$ then $\operatorname{deg}_{U}(w)=0$ and $\operatorname{lead}(w)=w$. If $\operatorname{deg}_{Y}(\pi(w))>0$ let

$$
\operatorname{lead}(w)=x_{1}^{a_{1}} \cdots x_{k}^{a_{k}} y_{k}^{b_{k}} \cdots x_{n}^{a_{n}} y_{n}^{b_{n}}
$$

where $k$ is the smallest number for which $b_{k} \neq 0$. Hence $w$ contains $u_{i k}$ for some $i<k$. Choose the largest number $j$ for which $w$ contains $u_{j k}$. Then $j<k$ and must be the largest among these numbers with $a_{j} \neq 0$.

Indeed, $w$ is a normal monomial. Assume that exists an $l$ for which $a_{l} \neq 0$ and $j<l<k$. Then $w$ cannot contain $x_{l}$ since $x_{l}$ is covered by $u_{j k}$. But it also cannot contain $u_{l m}$ since $m \geq k$ by the choice of $k$ and if $m=k$ then $w$ contains $u_{l k}$ contrary to the choice of $j$; so $m>k$ and $u_{j k}$ and $u_{l m}$ would intersect each other.

Therefore $w=u_{j k} w_{1}$ where $u_{j k}$ is uniquely determined by $w$. By induction lead $\left(w_{1}\right)$ determines $w_{1}$ and so $w$ is determined by lead $(w)$. This, of course, implies that lead $(w)$ are different for different normal monomials and hence the images $\pi(w)$ of these monomials are linearly independent.

\section{ACKNOWLEDGEMENTS}

The first author is grateful to the Department of Mathematics of the Wayne State University in Detroit for the warm hospitality during his visit when most of this work was carried out.

\section{REFERENCES}

[AL] W.W. Adams, P. Loustaunau, An Introduction to Gröbner Bases, Graduate Studies in Math. 3, AMS, Providence, R.I., 1994.

[E] A. van den Essen, Polynomial Automorphisms and the Jacobian Conjecture, Progress in Math., Birkhauser, 2000.

[F] G. Freudenburg, Algebraic Theory of Locally Nilpotent Derivations, Encyclopaedia of Mathematical Sciences, 136, Invariant Theory and Algebraic Transformation Groups, VII. Springer-Verlag, Berlin, 2006.

[K] J. Khoury, Locally Nilpotent Derivations and Their Rings of Constants, Ph.D. thesis, Univ. Ottawa, 2004

[N] A. Nowicki, Polynomial Derivations and Their Rings of Constants, Uniwersytet Mikolaja Kopernika, Torun, 1994. Available at: http://www-users.mat.uni.torun.pl/ anow/polder.html

[S] C.S. Seshadri, On a theorem of Weitzenböck in invariant theory, J. Math. Kyoto Univ. 1 (1962), 403-409.

[T] A. Tyc, An elementary proof of the Weitzenböck theorem, Colloq. Math. 78 (1998), 123-132.

[W] R. Weitzenböck, Über die Invarianten von linearen Gruppen, Acta Math. 58 (1932), 231-293.

Institute of Mathematics and Informatics, Bulgarian Academy of Sciences, 1113 SOFia, Bulgaria

E-mail address: drensky@math.bas.bg

Department of Mathematics, Wayne State University Detroit, Mi 48202, USA.

E-mail address: lml@math.wayne.edu 